\title{
Liste des membres
}

Volume 36, 1969

URI : https://id.erudit.org/iderudit/1007300ar

DOI : https://doi.org/10.7202/1007300ar

Aller au sommaire du numéro

Éditeur(s)

Les Éditions Historia Ecclesiæ Catholicæ Canadensis Inc.

ISSN

0318-6172 (imprimé)

1927-7067 (numérique)

Découvrir la revue

Citer ce document

(1969). Liste des membres. Sessions d'étude - Société canadienne d'histoire de

l'Église catholique, 36, 101-110. https://doi.org/10.7202/1007300ar

Tous droits réservés @ Les Éditions Historia Ecclesiæ Catholicæ Canadensis Inc., 1970
Ce document est protégé par la loi sur le droit d'auteur. L'utilisation des services d'Érudit (y compris la reproduction) est assujettie à sa politique d'utilisation que vous pouvez consulter en ligne.

https://apropos.erudit.org/fr/usagers/politique-dutilisation/ 
SOCIÉTÉ CANADIENNE D'HISTOIRE DE L'ÉGLISE CATHOLIQUE

\section{MEMBRES BIENFAITEURS *}

Veuillez informer le secrétaire de tout changement d'adresse

1942 - Son Excellence $M^{\mathrm{gr}}$ Arthur Douville, Evêque de Saint-Hyacinthe, Evêché de Saint-Hyacinthe, Saint-Hyacinthe, P.Q.

1944 - M. le chanoine Robert Charland, Séminaire de Nicolet, Nicolet, P.Q.

1945 - Le très révérend Père Provincial des Missionnaires Oblats de Marie-Immaculée, 3456, avenue Ontario, Montréal 25, P.Q.

$1946-\mathrm{M}^{\mathrm{gr}}$ Adrien Verrette, P.D., 517, rue Pine, Manchester, N.H., E.-U.

1949 - M. J.-E. Guertin.

1958 - Son Excellence $\mathbf{M}^{\text {gr }}$ Gérard-Marie Coderre, Evêque de Saint-Jean, Saint-Jean, P.Q.

\section{MEMBRES DONATEURS *}

1945 - M. Conrad Charlebois, C.O.St-G.G., 70, rue Maurice, Hull, P.Q.

194.5 - M. C.-J. Hubert, 1490, avenue Laframboise, Saint-Hyacinthe, P.Q.

1945 - M. le major Gustave Lanctot, 5642, avenue Woodbury, Montréal 250, P.Q.

1945 - La révérende Mère Provinciale des Sours de la Présentation, Saint-Hyacinthe, P.Q.

1945 - $\mathrm{M}^{\mathrm{gr}}$ Albert Tessier, Séminaire des Trois-Rivières, Trois-Rivières, P.Q.

1945 - Le très révérend Père Recteur de l'Université d'Ottawa, Ottawa, Ont.

1949 - Son Excellence $\mathrm{M}^{\mathrm{gr}}$ Georges Cabana, 104 est, rue Sherbrooke, Montréal 18, P.Q.

1949 - M. C.-R. Dufault, 505, rue Langevin, Saint-Boniface, Man.

1953 - M. Gustave Bellefleur, 3973, rue Mentana, Montréal 132, P.Q.

1958 - Son Excellence $\mathrm{M}^{\mathrm{gr}}$ Charles-Eugène Parent, Archevêché de Rimouski, Rimouski, P.Q.

1958 - Sœurs de la Charité, La supérieure générale des, Hôtel-Dieu, Saint-Hyacinthe, P.Q.

1963 - M. l'abbé Charles Michaud, Maison mère des Sœurs du SaintRosaire, Rimouski, P.Q.

1964-Cournoyer, M. l'abbé Georges-Henri, Presbytère Saint-Joseph, Saint-Hyacinthe, P.Q.

1964 - Tremblay, $\mathrm{M}^{\mathrm{gr}}$ Victor, P.D., Petit Séminaire, Chicoutimi, P.Q.

* On peut devenir bienfaiteur en versant la somme de cent dollars, membre donateur en versant cinquante dollars. 


\section{MEMBRES}

Abbaye de Notre-Dame de Nazareth, L', Rougemont, P.Q.

Académie de Québec, La Bibliothèque de l', 2410, chemin Sainte-Foy, Québec 10, P.Q.

Agnès-de-Jésus, n.d.b.c., Rév. Sœur, Ecole Sainte-Claire, ChicoutimiNord, P.Q.

Allaire, Rév. Père G.-H., c.s.v., 1145, avenue Saint-Viateur, Outremont 153, P.Q.

American Bibliographical Center, 800 Last Micheitorena STt, Santa Barbara, California, U.S.A.

Anne-Marie, n.d.b.c., Rév. Sœur, Maison-Mère du Bon Conseil, Chicoutimi, P.Q.

Archambault, M. le docteur A.-D., 4600, boulevard LaSalle, Verdun 203, P.Q.

Archives acadiennes, Bibliothèque générale, Université de Moncton, Moncton, N.-B.

Archives du Monastère, Les Hospitalières de Saint-Augustin, 75, rue des Remparts, Québec 4, P.Q.

Archives Publiques du Canada, La Bibliothèque des, 395, rue Wellington, Ottawa 4, Ont.

Archiviste du Québec, L', Parc des Champs de Bataille, Québec 6, P.Q. Babin, M. Roland, 620, avenue E, Murdockville, P.Q.

Baillargeon, M. l'abbé Noël, Séminaire de Québec, C.P. 460, Québec, P.Q. Baudry, Le R.P. René, c.s.c., Ambassade du Canada, 35, avenue Montaigne, Paris VIII ${ }^{e}$, France.

Beaudin, M. l'abbé François, 2000 ouest, rue Sherbrooke, Montréal 109, P.Q.

Beaudry, Rev. A.-T., 50 Melville, Pittsfield, Mass., U.S.A.

Beaulieu, M. Gaston, 37 Valentine Drive, Don Mills, Ont.

Beaulieu, M. le chanoine Henri, Séminaire de Québec, C.P. 460, Québec, P.Q.

Beaulieu, M. Wilfrid, Le Travailleur, C.P. 195, Worchester 1, Mass., U.S.A.

Behrer, M. W., C.R., Président, Commission des Ecoles catholiques, 1460, chemin Sainte-Foy, Québec 6, P.Q.

Bélanger, $\mathrm{M}^{110}$ Françoise, 5373, rue Durocher, Outremont 153, P.Q.

Bélanger, $\mathbf{M}^{\mathrm{gr}}$ Léon, P.D., curé, l'Islet, P.Q.

Bélanger, $\mathrm{M}^{\mathrm{gr}}$ René, P.D., 980, rue Richelieu, Québec, P.Q.

Bélanger, Son Excellence $\mathbf{M}^{\mathrm{gr}}$ Valérien, 1071, rue Cathédrale, Montréal 3, P.Q.

Bertrand, l'honorable M. Lionel, C.P. 190, Sainte-Thérèse-de-Blainville, P.Q.

Bérubé, M. le chanoine Léo, Archevêché de Rimouski, Rimouski, P.Q.

Bibeau, R.P. Hector, s.m.m., Séminaire Montfort, C.P. 115, Papineauville, P.Q.

Biblioteca Apostolica Vaticana, Cité du Vatican.

Bibliothécaire, Le R.P., Couvent des Pères Dominicains, 96, avenue Empress, Ottawa 4, Ont.

Bibliothécaire, Le R.P., Abbaye Saint-Benoît-du-Lac. P.Q. 
Bibliothèque, Collège Basile-Moreau, 845, rue Sainte-Croix, Montréal 381, P.Q.

Bibliothèque, Collège de Saint-Boniface, 200, avenue de la Cathédrale, Saint-Boniface 6, Man.

Bibliothèque, Université Laurentienne, Sudbury, Ont.

Bibliothèque de l'Assemblée législative de la Province de Québec, La, Hôtel du Gouvernement, Québec, P.Q.

Bibliothèque de la ville de Montréal, La, 1210 est, rue Sherbrooke, Montréal 110, P.Q.

Bibliothèque du Parlement, La, Hôtel du Gouvernement, Ottawa, Ont. Bibliothèque Nationale, La, 1700, rue Saint-Denis, Montréal 129, P.Q. Bibliothèque des Trois-Rivières, C.P. 1207, 1220, rue Jean-Nicolet, Trois-Rivières, P.Q.

Bibliothèque du Séminaire, C.P. 410, Joliette, P.Q.

Bibliothèque du Séminaire de Sherbrooke, C.P. 790, rue Marquette, Sherbrooke, P.Q.

Billy, M. J.-Louis, C.O.St-G.G., 425, rue Saint-Patrice, Ottawa 1, Ont. Bonenfant, $\mathrm{M}^{11 \mathrm{e}}$ Raymonde, 874, avenue Royale, St-Jean d'Orléans (Cté Montmorency), P.Q.

Boucher, Rév. Sœur Marie-Reine, s.c.l.m., Maison Provinciale du BonPasteur, 545, rue Saint-Amable, Québec, P.Q.

Bourassa, M. le curé Lionel, Lavigne (Nipissing), Ont.

Bourgeois, $M^{110}$ Marguerite-R., 74, rue Notre-Dame, app. 2, Hull, P.Q. Brouillard, $\mathrm{M}^{\mathrm{gr}}$ Armand, P.D., Séminaire, C.P. 370, Saint-Hyacinthe, P.Q. Bruchési, Son Excellence M. Jean, app. 901, 2 Square Westmount, boul. de Maisonneuve, Westmount, P.Q.

Bureau des Archives, Sœurs de l'Assomption de la Sainte-Vierge, Nicolet, P.Q.

Campeau, M. le curé O., Hanmer, Ont.

Capucins, Les révérends Pères, $460,8^{\bullet}$ avenue, Québec 3, P.Q.

Carleton University, The Library, Colonel By Drive, Ottawa, Ont.

Carrière, Le R.P. Gaston, o.m.i., Séminaire universitaire, 249, rue Main, Ottawa, Ont.

Cartier, M. le curé Lucien, Saint-Aimé (Richelieu), P.Q.

Charbonneau, M. Louis, 1, chemin Beckwith, Ottawa, Ont.

Charbonneau, M. Rhéal, Secrétaire national de l'Action catholique canadienne, 3827, rue Saint-Hubert, Montréal, P.Q.

Charron, M. le curé Léo, Pain Court, Ont.

Chartrand, M. le curé Roland, 369, Grande Côte, Sainte-Thérèse-Ouest, Terrebonne, P.Q.

Chaussé, R.P. Gilles, s.j., Collège Brébeuf, 32 chemin Sainte-Catherine, Montréal 250, P.Q.

Clercs de Saint-Viateur, La Maison Provinciale des, a/s Le Bibliothécaire, 1145, rue Saint-Viateur, Outremont 153, P.Q.

Collège Bourget, La Bibliothèque, Rigaud, P.Q.

Collège des Jésuites, Le, 1150 ouest, rue Saint-Cyrille, Québec 6, P.Q.

Collège de Jésus-Marie de Sillery, Le, 2047, chemin Saint-Louis, Québec 6, P.Q.

Collège de Lévis, $M^{\text {gr }}$ le Supérieur du, Lévis, P.Q. 
Collège Sainte-Foy, La Bibliothèque, 2410, chemin Sainte-Foy, Québec 10, P.Q.

Collège Saint-Laurent, a/s R.P. Bibliothécaire, 625, boulevard SainteCroix, Ville Saint-Laurent, Montréal 381, P.Q.

Collège Sainte-Anne-de-la-Pocatière, La Bibliothèque du, Sainte-Anne-dela-Pocatière, P.Q.

Collège Saint-Maurice, La Directrice du, 640, rue Girouard, SaintHyacinthe, P.Q.

Compagnie de Ísuss, Le Révérend Père Provincial de la, 3215, chemin Sainte-Catherine, Montréal 250, P.Q.

Comptois, M. le docteur B., 36, rue Elm est, Sudbury, Ont.

Conférence Catholique Canadienne, M. le Secrétaire de la, 90, rue Parent, Ottawa, Ont.

Congrégation de Notre-Dame, Le Bureau des Archives de la, 3040 ouest, rue Sherbrooke, Montréal 215, P.Q.

Cormier, Le R.P. Clément, c.s.c., Université de Moncton, Moncton, N.-B.

Corriveau, M. l'abbé Robert, Napierville (Comté Napierville), P.Q.

Cossette, Le R.P. Joseph, s.j., archiviste, C.P. 130, Saint-Jérôme, P.Q.

Cournoyer, M. l'abbé Georges-Henri, 886, rue Saint-Pierre, Saint-Rochsur-Richelieu, P.Q.

Crète, Rév. Père Florian, c.s.v., C.P. 279, Rigaud-de-Vaudreuil, P.Q.

Dansereau, M. Antoine, p.s.s., Collège de Montréal, 1931 ouest, rue Sherbrooke, Montréal 109, P.Q.

DeBlois, M. le curé Alexandre, V.F., Sainte-Marie-de-Beauce, P.Q.

Décosse, Son Excellence $\mathrm{M}^{\mathrm{gr}}$ Aimé, Evêque de Gravelbourg, Gravelbourg, Sask.

De Lagrave, é.c., M. François, 2400, chemin Sainte-Foy, Québec 10, P.Q.

Delisle, Solange, 28 est, rue des Bouleaux, Québec, P.Q.

DeMoissac, La révérende Sœur E., s.g.m., 51, rue Despins, SaintBoniface 5, Man.

Deschâtelets, Le T.R.P. Léo, o.m.i., Supérieur général, 290, via Aurelia, Rome, Italie.

Desjardins, M. Gérard, 281, rue Beaubassin, Saint-Anselme, N.-B.

Desjardins, Le révérend Père Paul, s.j., C.P. 130, Saint-Jérôme (Comté Terrebonne), P.Q.

Desjardins, Le R.P. Léopold, c.ss.r., Mont Saint-Joseph, 161, rue Principale, Aylmer-Est, P.Q.

Dionne, M. J.A., ingénieur professionnel, 760, avenue Wiseman, Outremont 153, P.Q.

Dominicains, Le Bibliothécaire du monastère des révérends Pères, 175 ouest, Grande Allée, Québec 6, P.Q.

Dominicains, Le couvent des révérends Pères, 5375, avenue Notre-Damede-Grâce, Montréal 260, P.Q.

Dorge, M. Lionel, C.P. 125, Saint-Norbert, Man.

Douville, M. Raymond, Secrétariat de la Province, 1105, avenue Belvédère, app. 221, Québec 6, P.Q.

Doyon, ${ }^{\text {gr }}$ Paul-Emile, P.A., V.G., Evêché, C.P. 879, Trois-Rivières, P.Q.

Drolet, M. Antonio, 740, rue Monk, Québec 6, P.Q. 
Drolet, M. Jean-Claude, a/s Séminaire de Chicoutimi, rue JacquesCartier, Chicoutimi, P.Q.

Dumais, M. le chanoine Charles, curé, C.P. 666, Sainte-Anne-de-laPocatière, P.Q.

Dumas, R.P. Réal, 4373, avenue de l'Esplanade. Montréal 131, P.Q.

Dumas, M. J.-E., 40 St. Mary's Road, Saint-Boniface, Man.

Dumouchel, M. le docteur Antoine, 1232 Stratford Road, Kansas City, Missouri, 64113, U.S.A.

Dupont, $\mathrm{M}^{\mathrm{e}}$ J.-E., Napierville, P.Q.

Durocher, M. René, Département d'Histoire, Université de Montréal. C.P. 6128, Montréal, P.Q.

Ecole Normale de Nicolet, La Sœur Directrice de l', Nicolet, P.Q.

Falardeau, M. l'abbé A., $7091-10^{\circ}$ Avenue, Ville Saint-Michel, Montréal 453, P.Q.

Filles de la Charité du Sacré-Cœur de Jésus, Les, a/s Sœur Rachel de Marie, 605 sud, avenue Bowen, Sherbrooke, P.Q.

Forest, M. Georges-J., 160, rue Marion, Saint-Boniface, Man.

Fortin, $\mathbf{M}^{\mathrm{gr}}$ Alphonse, P.D., Séminaire de Rimouski, Rimouski, P.Q.

Fournier, $D^{r}$ Robert, 287, avenue Saint-Jérôme, Matane, P.Q.

Foyer de Saint-Célestin, Saint-Célestin (Nicolet), P.Q.

Franciscains, Les révérends Pères, 5750, boulevard Rosemont, Montréal 410, P.Q.

Franciscains, La Bibliothèque des, 2010 ouest, boulevard Dorchester, Montréal 108, P.Q.

François-Xavier, o.s.a., Rév. Sœur, Hôtel-Dieu, Alma, P.Q.

Frenette, Son Excellence $\mathbf{M}^{\text {gr }}$ Emilien, Evêque de Saint-Jérôme, SaintJérôme, P.Q.

Frigon, Rév. Sœur Thérèse, f.c.s.p., 5655, rue de Salaberry, Montréal 390, P.Q.

Gagné, M. l'abbé A., Archevêché de Québec, C.P. 459, Québec 4, P.Q.

Gagné, $M^{\text {me }}$ Léo, Saint-Tharsicius (Matapédia), P.Q.

Gagnon, $\mathrm{M}^{\mathrm{gr}}$ Antoine, P.A., Institut de Technologie, 25, rue Saint-Louis, Rimouski, P.Q.

Gagnon, M. l'abbé François, Collège Sainte-Anne-de-la-Pocatière, SainteAnne-de-la-Pocatière, P.Q.

Galarneau, M. Claude, 175, avenue Laurier, Québec 4, P.Q.

Gallant, Le R.P. Arthur, c.j.m., Paroisse Sainte-Amélie, C.P. 460, Baie Comeau, P.Q.

Gariépy, M. l'abbé Gérard, Berthier-en-Bas (Montmagny), P.Q.

Gaudron, R.P. E., o.f.m., Culture, 733, rue de l'Alverne, Québec, P.Q.

Gauthier, M. le docteur Dominique, Shippagan, N.-B.

Gauthier, Le R.P. Léandre, o.m.i., C.P. 361, Fort-Qu'Appelle, Sask.

Gauthier, $M^{\mathrm{m}}$ Paul-R., 605, $8^{\mathrm{e}}$ Avenue, Québec 5, P.Q.

Gélineau, $\mathrm{M}^{\mathrm{gr}}$ J.-Napoléon, P.D., V.G., curé de la cathédrale, 60 , rue Guigues, Ottawa 2, Ont.

Gendron, M. l'abbé Paul, Sainte-Clotilde (Châteauguay), P.Q.

Geoffroy, M. l'abbé J.-Hector, curé, Saint-Félix-de-Valois (Joliette), P.Q.

Germain, $M^{e}$ Stanislas, 7l, rue Saint-Pierre, Québec 2, P.Q. 
Gervais, Le R.P. Emile, s.j., Maison Bellarmin, 25 ouest, rue Jarry, Montréal 351, P.Q.

Giard, M. Gilles, nolaire, 1555, rue Girouard, Saint-Hyacinthe, P.Q.

Giguère, R.P. Georges-Emile, s.j., 25 ouest, rue Jarry, Montréal 351, P.Q. Gilbert, M. Rémi-Nelson, C.A., C.P. 621 (Hauteville), Québec 4, P.Q. Gingras, $\mathrm{M}^{\mathrm{me}}$ Marcelle-G., 470, chemin Saint-Louis, Québec 6, P.Q.

Gosselin, Rév. Frère Daniel, é.c., 1049, route de l'Eglise, Sainte-Foy, Québec 10, P.Q.

Gosselin, M ${ }^{\text {gr }}$ Paul-Emile, P.D., 47, rue des Remparts, Québec 4, P.Q.

Goulet, M. le curé Philibert, Saint-Gervais (Bellechasse), P.Q.

Goyette, M. Armand. 19, boulevard des Erables, Iberville, P.Q.

Grand'Maison, Rév. Sœur Georgette, r.s.r., 280, avenue Belzile, Rimouski, P.Q.

Grand Séminaire de Québec, M. le Bibliothécaire, Québec 10. P.Q.

Gravel, M. l'abbé Henri, 1500, rue Orléans, Montréal 403, P.Q.

Grisé, Rév. Père Jacques, c.s.c., 1330, chemin Sainte-Foy, Québec 6, P.Q.

Hanzéli, M. Victor-E., 41l N.E. 81st Street. Seattle, Washington, 98105. U.S.A.

Hardy, M. René, 4187, rue Louis-Pinard. Trois-Rivières, P.Q.

Harvard College Library, Record Serials, Cambridge, Mass., U.S.A., 02138.

Héroux, Rév. Sœur Liliane. Les RR.SS. de la Charité de Québec, rue D'Estimauville, Québec, P.Q.

Hôpital général de Verdun, L', 4000, boulevard La Salle. Verdun, Montréal 203, P.Q.

Hudon, M. Jean-Claude, 209, rue Bossé, Chicoutimi, P.Q.

Hurtubise, R.P. Pierre, o.m.i., 249, rue Main, Ottawa, Ont.

Institution des Sourdes-Muettes, L', 3725, rue Saint-Denis, Montréal 103, P.Q.

Jean, Rév. Søur Marguerite, 1080, rue Lachevrotière, Québec, P.Q.

Jésuites, Les révérends Pères, Collège de Saint-Boniface, 200, avenue de la Cathédrale, Saint-Boniface 6, Man.

Jésuites, Les révérends Pères, 14, rue Dauphine, Québec 4, P.Q.

Joseph-Adonis, R.F., f.m.s., Scolasticat central de Montréal, 7000, rue Marie-Victorin, Montréal 4.62, P.Q.

Lachance, M. André, Département d'Histoire, Université de Sherbrooke, Sherbrooke, P.Q.

Lacourcière, M. Luc, Université Laval, Québec 10, P.Q.

Lacroix, Rév. Père Benoît, o.p., 2715, chemin Sainte-Catherine, Montréal 250, P.Q.

Lamirande, R.P. Emilien, o.m.i., Scolasticat Saint-Joseph, 185, rue Main, Ottawa, Ont.

Landry, Rév. Père Thomas-Marie, o.p., 2490, avenue Royale, Courville. Québec 5, P.Q.

Langlois, $\mathrm{M}^{\mathrm{gr}}$ W.-J., P.D., V.F., St. Mary's Academy, Windsor. Ont.

Larouche, M. Georges-G., 2074, avenue Royale, Courville, Québec 5, P.Q.

Latourelle, $\mathrm{M}^{\mathrm{gr}}$ le curé J.-A., P.D., C.P. 740, Temiskaming. P.Q.

Lavallée, M. l'abbé Germain, C.P. 790, Sherbrooke, P.Q. 
LeBlanc, Son Excellence $\mathbf{M}^{\mathrm{gr}}$ Camille-André, Evêque de Bathurst, C.P. 4.60, Bathurst, N.-B.

Leblanc, $\mathrm{M}^{\circ}$ Lionel, Protonotaire de la Cour supérieure, Palais de Justice, Saint-Hyacinthe, P.Q.

LeBlond, M. le docteur Sylvio, 341, avenue du Séminaire, Chicoutimi, P.Q.

Leclaire, $\mathrm{M}^{\mathrm{gr}}$ J.-Charles, P.D., V.G., C.P. 218, Sorel, P.Q.

Leclerc, R.P. Jean, s.j., Université de Sudbury, Sudbury, Ont.

Lefebvre, Rév. Søur Estelle, Hôtel-Dieu, 3840, rue Saint-Urbain, Montréal 13l, P.Q.

Lefebvre, Jean-Jacques, archiviste en chef, Cour supérieure, Palais de Justice, Montréal, P.Q.

Le Moignan, M. l'abbé Michel, curé, Cathédrale du Christ-Roi, C.P. 159, Gaspé, P.Q.

Lemoyne, $\mathrm{M}^{11 \mathrm{e}}$ Georgette, 3950, Parc Lafontaine, Montréal 132. P.Q.

Lépine. M. Léopold, 715, rue Saint-Vallier, Québec 2, P.Q.

Lessard, M. le chanoine Léon-N., Collège de Lévis, Lévis, P.Q.

Letendre, M. J.-Antoine, Archiviste, Evêché de Nicolet, Nicolet, P.Q.

Letendre, M. le chanoine J.-G., Assistant-procureur, Archevêché de Sherbrooke, 130, rue de la Cathédrale, C.P. 430, Sherbrooke, P.Q.

Létourneau, M. Claude, 115, rue Carillon, Hull, P.Q.

Levesque, Son Excellence $\mathbf{M}^{\mathrm{gr}}$ Louis, Archevêque de Rimouski, Rimouski, P.Q.

Lottinville, M. Wilfrid Lemaistre de, 494, rue Fraser, Québec 6, P.Q.

Madeleine-Durand, Rév. Sœur, f.c.s.p., Annaliste, Providence maisonmère, 5655, rue de Salaberry, Montréal 390, P.Q.

Madore, M ${ }^{110}$ Marie-Ange, 853 est, rue Sherbrooke, Montréal 132, P.Q.

Maison Provinciale Sainte-Marie, Rév. Søurs Grises, 86, rue Ignace, Sudbury, Ont.

Malouin, $\mathrm{M}^{\mathrm{me}}$ Reine, 1817, rue des Intendants, Québec 3, P.Q.

Mantha, M. Hector, 65, rue Milton, Montréal 130, P.Q.

Maranda, Adéline, Foyer Saint-Tite-des-Caps (Montmorency), P.Q.

Marie-de-la-Nativité, o.s.u., Rév. Mère, Monastère des Ursulines, C.P. 820, Roberval, P.Q.

Marie-Jeanne-de-Domrémy, Rév。 Sœur, Ecole Normale, Saint-Jérôme, P.Q.

Marion, M. Séraphin, 131, rue Sunnyside, Ottawa, Ont.

Martin, Son Excellence $\mathbf{M}^{\mathrm{gr}}$ Albertus, Evêché de Nicolet, Nicolet, P.Q.

Mathieu, M. le curé Aimé, 7075, route Marie-Victorin, Tracy, P.Q.

Mayrand, $\mathbf{M}^{\mathrm{gr}}$ Paul, P.A., Evêché, Nicolet, P.Q.

Michaud, M. l'abbé Charles, Maison-Mère des Sœurs du Saint-Rosaire, Rimouski, P.Q.

Missionnaires de l'Immaculée-Conception, Les Sœurs, Ecole secondaire Immaculée-Conception, 60, rue Saint-Joseph, Granby, P.Q.

Missionnaires de l'Immaculée-Conception, Les Sœurs, 906, rue Syndenham, Chicoutimi, P.Q.

Missionnaires Oblats de Marie-Immaculée, Noviciat Notre-Dame, Richelieu, P.Q. 
Monastère du Précieux-Sang, La Supérieure du, C.P. 186, Saint-Hyacinthe, P.Q.

Morisset, M. Fennand, 181, iue d'Youville, Sudburry, Ont.

Normand, $\mathbf{M}^{\mathrm{me}}$ Maurice, 1120, René-Kimber, app. 4, Pavillon de Normandville, Trois-Rivières, P.Q.

Nourry, Le R.P. L.-M., s.m.m., 1265, rue Wyld, C.P. 718, North Bay, Ont.

O'Bready, $\mathrm{M}^{\mathrm{gr}}$ Maurice, P.D., Ecole Normale de L'Estrie, rue Jolliet, Sherbrooke, P.Q.

Oblats de Marie-Immaculée, Rév. Père Provincial des, 3390, chemin Saint-Louis, Québec 10, P.Q.

Oss, $M^{\mathrm{me}}$ Esther-Y., 1278, rue Lemoine, Sillery, Québec 6, P.Q.

Panneton, M. le chanoine G., Couvent de Marie-Réparatrice, 2975, boulevard Laviolette, Trois-Rivières, P.Q.

Paré, Son Excellence $M^{g r}$ Marius, C.P. 278, Evêché de Chicoutimi, Chicoutimi, P.Q.

Parent, $\mathrm{M}^{11 \mathrm{e}}$ Corinne-Hélène, 215 ouest, rue Somerset, Ottawa, Ont.

Parenteau, $\mathrm{M}^{\mathrm{gr}}$ Antonio, P.D., 155, rue Saint-Marcel, Drummondville. P.Q.

Petit Séminaire de Chicoutimi, La Bibliothèque du, rue Jacques-Cartier, Chicoutimi, P.Q.

Picard, M. Jean-Théo., 219, rue Saint-Michel, Saint-Jean-sur-Richelieu, P.Q.

Plante, M. l'abbé Hermann, Séminaire Saint-Joseph, Trois-Rivières, P.Q.

Potvin, M. l'abbé Pascal, 53, rue Déziel, Lévis, P.Q.

Pouliot, Le R.P. Léon, s.j., C.P. 130, Saint-Jérôme, P.Q.

Présentation de Marie, La Supérieure provinciale des Sours de la, 650 , rue Girouard, Saint-Hyacinthe, P.Q.

Présentation de Marie, Les Sœurs de la, C.P. 134, Acton Vale, Bagot, P.Q.

Prévost, M. Edouard, 1540, avenue Bernard, app. 29, Outremont, Montréal 153, P.Q.

Prince, $\mathrm{M}^{\mathrm{me}}$ Berthe, C.P. 231, Sainte-Thérèse (Terrebonne), P.Q.

Proulx, Son Exc. $\mathrm{M}^{\mathrm{gr}}$ A., Evêque d'Alexandria, Alexandria, Ont.

Providence, La maison mère des Sœurs de la, 12055, rue Grenet, Montréal 390, P.Q.

Provinciale, Rév. Mère, s.g.m., 151, rue Despins, Saint-Boniface, Man.

Provost, M. l'abbé Honorius, Séminaire de Québec, Québec, P.Q.

Raymond, M. le chanoine C.-Eugène, Institut Chanoine-Beaudet, SaintPascal-de-Kamouraska, P.Q.

Raymond, M. l'abbé Marius, Presbytère Saint-Norbert, Rimouski, P.Q.

Raymond, M. Raoul, 8960, rue Saint-Denis, Montréal 354, P.Q.

Rédemptoristes, Le R.P. Recteur du monastère des, 871, rue Ontario. Sherbrooke, P.Q.

Rédemptoristes, Le Supérieur des Pères, Sainte-Anne-de-Beaupré, P.Q.

Rédemptoristes, Les révérends Pères, Sainte-Anne-des-Chênes, Man.

Renaud, Rév. Sœur Rose-Anne, Maison Provinciale du Bon-Pasteur, 424, rue Bégin, Chicoutimi, P.Q.

Robitaille, M. l'abbé Léger, 357, boul. Valcartier, Loretteville, P.Q.

Rodrigue, $\mathrm{M}^{\mathrm{gr}}$ Louis-Joseph, P.D., 202, rue de la Princesse, Lachute (Argenteuil), P.Q. 
Rollin, M. l'abbé Donat, 253, boul. Saint-Joseph, Hull, P.Q.

Routhier, Son Excellence $\mathrm{M}^{\mathrm{gr}}$ Henri, o.m.i., McLennan, Alta.

Roux. $\mathrm{M}^{\mathrm{gr}}$ le curé Alphonse, 245 est, rue Notre-Dame, Victoriaville, P.Q.

Roy, M. Antoine, 165, Grande-Allée, Québec 4, P.Q.

Sabourin, $\mathbf{M}^{\circ}$ Roland, 370, rue Champlain, C.P. 186, Saint-Jean, P.Q.

Saint-Amour, Rév. Sœur Marie-Reine, 214, rue Notre-Dame, Rouyn, P.Q.

Saint-Boniface, M. le Bibliothécaire de l'archevêché de, Saint-Boniface, Man.

Saint-Germain, M. Yves, Département d'Histoire, Faculté des Lettres,

Université de Montréal, C.P. 6128, Montréal 250, P.Q.

Saint-Jean, M. et $\mathrm{M}^{\mathrm{me}}$ Albert, 1028, $2^{\mathrm{e}}$ Avenue (Verdun), Montréal 203, P.Q.

Saint-Jean, CEGEP de, C.P. 310, Saint-Jean, P.Q.

Saint-Pierre, M. René, 3095, rue Girouard, Saint-Hyacinthe, P.Q.

Saint-Sacrement, Les Pères du Très, 4450, rue Saint-Hubert, Montréal 176, P.Q.

Sainte-Germaine, s.c.o., Rév. Sœur, Supérieure, Hôpital Saint-Joseph, rue Mackenzie, Sudbury, Ont.

Sainte-Theresita, s.c.i.m., Rév. Sœur, Collège du Bon-Pasteur, avenue Lafontaine, Chicoutimi, P.Q.

Saucier, M. le docteur Roland, C.P. 203, Chicoutimi, P.Q.

Sauvageau, Dom Fidèle, o.c.s.o., Abbaye Cistercienne Notre-Dame-duLac, La Trappe, P.Q.

Savard, $M^{11 e}$ Blanche-Alice, 21 ouest, rue Price, Chicoutimi, P.Q.

Savard, M. Pierre, 2607, rue Père-Druillettes, Québec 10, P.Q.

Scolasticat Central de Montréal, 7000, rue Marie-Victorin, Montréal 476, P.Q.

Scolasticat Saint-Charles, 455, boulevard Querbes, Joliette, P.Q.

Secrétaire générale, s.g.m., Rév. Mère, 9409 ouest, boul. Gouin, Pierrefonds, P.Q.

Séminaire de Nicolet, La Bibliothèque, Nicolet, P.Q.

Séminaire du Sacré-Cour, La Bibliothèque, Station Saint-Victor (Beauce), P.Q.

Séminaire de Saint-Hyacinthe, M. le Bibliothécaire, C.P. 370, SaintHyacinthe, P.Q.

Séminaire des Missions Etrangères, 60, rue Desnoyers, Pont-Viau, Montréal 207, P.Q.

Séminaire Saint-Thomas, M. le Bibliothécaire du, Valleyfield, P.Q.

Séminaire des Trois-Rivières, M. le Bibliothécaire du, Trois-Rivières, P.Q.

Sergerie, $M^{\mathrm{me}}$ Adéla, 14748 est, boul. Gouin, Rivière-des-Prairies, Montréal $478, P . Q$.

Simard, R.P. André, c.ss.r., Paroisse du Perpétuel Secours, 132, rue Blake, Hamilton, Ont.

Simard, M. l'abbé Jean-Paul, Petit Séminaire, rue Jacques-Cartier, Chicoutimi, P.Q.

Société d'Histoire de l'lle-Jésus, La, 6715, rue Richelieu (Auteuil), Laval, P.Q. 
Société historique de la Côte-du-Sud, La, Collège Sainte-Anne, SainteAnne-de-la-Pocatière, P.Q.

Société historique de Québec, La, Séminaire de Québec, C.P. 460, Québec 4, P.Q.

Søurs de l'Assomption, La révérende Mère générale des, Nicolet, P.Q. Sœurs de la Charité de Québec, La révérende Mère générale des, avenue d'Estimauville, Québec 5, P.Q.

Sœurs de la Charité, Les, Hôtel-Dieu, Saint-Hyacinthe, P.Q.

Sœure de la Charité d'Qttawa, I'Arrhiviste des. 9. rue Bruyère, Ottawa, Ont.

Sœurs de Notre-Dame du Bon-Conseil, La Maîtresse générale des Etudes des, Maison mère, Chicoutimi, P.Q.

Sœurs de Sainte-Anne, La révérende Mère générale des, Mont SainteAnne, 1950, rue Provost, Montréal 402, P.Q.

Soeurs des Saints-Noms de Jésus-Marie, La Mère provinciale des, Académie Sainte-Marie, Windsor, Ont.

Soublière, M. Hector, 240, avenue Algonquin, North Bay, Ont.

Soucy, M. le chanoine Zénon, C.P. 8, Le Bic, P.Q.

State Historical Society, The, 816 State St., Madison 6, Wisconsin, U.S.A.

Sylvain, M. Philippe, Institut d'Histoire, Faculté des Lettres, Cité universitaire, Québec 10, P.Q.

Tardif, R.P. Emile, o.m.i., 9916 - 110 Rue, Edmonton, Alta.

Thibault, M. l'abbé Alfred, C.P. 153, Wottonville, P.Q.

Thibault, M. le chanoine Raoul, Maison-mère du Saint-Rosaire, Rimouski, P.Q.

Tremblay, M. Yves, 1140, rue Paul-VI, Charlesbourg, Québec 7, P.Q.

Trottier, Le R.P. Aimé, c.s.c., Oratoire Saint-Joseph, Montréal 26, P.Q.

Trudeau, Le R.P. Paul-Albert, c.s.v., 1145, rue Saint-Viateur, Outremont 153, P.Q.

Turgeon, $\mathrm{M}^{\mathrm{gr}}$ Emile, P.D., 980, rue Richelieu, Québec 4, P.Q.

Université Laval, Division Canadiana, Bibliothèque, Québec, P.Q.

University of Chicago, Chicago, Ill., U.S.A.

Université de Sudbury, Le R.P. Recteur de l', Sudbury, Ont.

Université d'Ottawa, La Bibliothèque, Ottawa 2, Ont.

Université Pontificale Grégorienne, La Bibliothèque, Piazza della Pilotta 4 , Rome, Italie.

Université Saint-Paul, La Bibliothèque, 233, rue Main, Ottawa, Ont.

Ursulines de Rimouski, Les, Rimouski, P.Q.

Ursulines de Roberval, Les, 712, boul. Saint-Joseph, Roberval, P.Q.

Vachon, M. André, 66, rue Pie-XII, Québec 10, P.Q.

Vachon, $\mathrm{M}^{\mathrm{gr}}$ Louis-Albert, Université Laval, Québec 15, P.Q.

Valois, $M^{\mathrm{gr}}$ Omer, P.D., V.G., Evêché, C.P. 470, Joliette, P.Q.

Vien, l'hon. Sénateur Thomas, suite 1506, Immeuble de la Banque Royale, 360 ouest, rue Saint-Jacques, Montréal 126, P.Q.

Vinet, M. Jean-Baptiste, p.s.s., 116 ouest, rue Notre-Dame, Montréal 126, P.Q.

Yelle, M. Gérard, p.s.s., Grand Séminaire, 2065 ouest, rue Sherbrooke. Montréal 109, P.Q. 